\title{
Der Rechtsgrund der Inanspruchnahme des Einen zugunsten des Anderen im sog. Aggressivnotstand"
}

\section{Luna Rösinger}

Warum darf eine Person in der Not in die Rechtssphäre einer anderen Person eingreifen, auch wenn letztere für die Gefahr in keiner Weise verantwortlich ist? Aus welchem Rechtsgrund dürfen Gefahren also gleichsam „kollektiviert“ (oder umverteilt) werden?

Diese Fragestellung, die hier anhand des sog. Aggressivnotstandes diskutiert werden soll, verspricht sowohl in Hinblick auf das Tagungsthema der „Kollektivierung“ als auch unter dem Aspekt der normentheoretischen Betrachtung des Strafrechts weiterführende Hinweise liefern zu können: Zum einen geht es materiell um das für die Notrechte unentbehrliche Verständnis des Einzelnen in der Gemeinschaft (dazu II. 1.). Zum anderen können zwei Legitimationsmodelle von Eingriffen aufgezeigt werden (dazu II. 2.-4.), deren Unterscheidung die Beurteilung der Rechtmäßigkeit eines Handelns im Aggressivnotstand vorstrukturieren kann (dazu III.). Zunächst erscheint jedoch eine kurze Erläuterung geboten, warum es überhaupt notwendig ist, in der Begründung des Instituts des Aggressivnotstandes einen Schritt zurückzugehen, wird der Weg doch allzu oft mit dem Verweis auf eine vermeintlich selbsterklärende Interessenabwägung abzukürzen gesucht (dazu I.)

\section{Kritik der Abwägungslehren nach dem Prinzip des überwiegenden Interesses}

Die Grundlage für den sog. Aggressivnotstand, der seinen positiv-rechtlichen Niederschlag in $\$ 34$ StGB sowie (beschränkt auf Sacheingriffe) in $\$ 904$ BGB gefunden hat, ${ }^{1}$ wird insbesondere im Prinzip des überwiegenden

* Der Beitrag wurde nach der Tagung angesichts der Corona-Pandemie geringfügig verändert.

1 Dagegen betrifft $₫ 228$ BGB den sog. Defensivnotstand, s. dazu noch unten II. 2. Eine dem $₫ 34$ StGB entsprechende Regelung findet sich in $₫ 16$ OWiG. 
Interesses gesucht. ${ }^{2}$ Danach muss ein minderwertiges Interesse zum Schutz eines überwiegenden Interesses geopfert werden, wenn es keine andere Möglichkeit zur Rettung des (wesentlich) wertvolleren Interesses gibt. Warum unter der Voraussetzung eines bestimmten Abwägungsverhältnisses der kollidierenden Interessen ein zur Förderung des einen Interesses geeigneter und erforderlicher Eingriff vorgenommen werden darf und das andere Interesse zurückstehen muss, ist damit freilich nicht erklärt. ${ }^{3}$ Eine Bewertung der betroffenen Interessen ist ohne Angabe eines haltbaren Prinzips der Vorrangentscheidung ${ }^{4}$ ebenso wenig möglich wie die Beantwortung der vorgelagerten Frage, warum die Freiheit des Einen zugunsten der Freiheit des Anderen überhaupt eingeschränkt werden können soll. Dies scheint jedoch gar nicht die Kernfrage des zugrunde liegenden Rechtsverständnisses zu sein: Es geht einer auf dem Prinzip des überwiegenden Interesses gründenden Rechtsordnung nicht um die Garantie von Freiheitsrechten, sondern darum, den Bestand an Gütern zu maximieren. ${ }^{5}$ Eine negative Abgrenzung von Freiheitssphären ist auch nicht mehr denkbar, wenn die ihr zugeordneten Güter und Interessen interpersonal zur Er-

2 So etwa bei Perron, in: Schönke/Schröder, Strafgesetzbuch, Kommentar, 30. Aufl. 2019, \34 Rn. 1, 22 ff.; Sternberg-Lieben, in: Schönke/Schröder, Strafgesetzbuch, Kommentar, 30. Aufl. 2019, Vor $\$ \$ 32$ Rn. 101. Siehe auch die Nachweise bei Helmers, Möglichkeit und Inhalt eines Notstandsrechts, 2016, S. 327 und - mit ausführlicher Darstellung der Entwicklung dieses Ansatzes - Pawlik, Der rechtfertigende Notstand. Zugleich ein Beitrag zum Problem strafrechtlicher Solidaritätspflichten, 2002, S. $32 \mathrm{ff}$.

3 Nach Köhler, ZStW 107 (1995), 10 (15) bezeichnet der - die Interessenabwägung enthaltende - Verhältnismäßigkeitsgrundsatz „allenfalls das Bestimmungsproblem. Eine begründete Lösung lässt er offen." Siehe auch die detaillierte Kritik bei Pawlik (Fn. 2), S. 39 ff., 48 ff. (zur herrschenden Gesamtabwägungslehre), insb. S. 50: „Hat man erst einmal die Entscheidungsregel von der Vorzugswürdigkeit des kleineren Übels akzeptiert, lässt sich die Frage, weshalb die Not des Einen überhaupt dazu in der Lage sein soll, das Recht des Anderen zu übertrumpfen, nicht einmal mehr sinnvoll stellen, geschweige denn beantworten." Siehe zur kritischen Auseinandersetzung mit dem Verhältnismäßigkeitsgrundsatz und dem Prinzip des überwiegenden Interesses im Kontext des Strafverfahrens Rösinger, Die Freiheit des Beschuldigten vom Zwang zur Selbstbelastung, 2019, S. $56 \mathrm{ff}$.

4 So die Kritik Köhlers an dem „Prinzip der Güterabwägung“ in ZStW 107 (1995), 10 (16).

5 Siehe die entsprechende Kritik bei Pawlik (Fn. 2), S. 43 f. Ähnlich die Kritik von Renzikowski, Notstand und Notwehr, 1994, S. 200, dass die „Individualgüter gerade in ihrer Zuordnung zu einer bestimmten Person rechtlichen Schutz" genießen und daher „nicht als ,Bilanzposten’ in der gesamten Güterwirtschaft der Gemeinschaft erscheinen“ können. 
zielung maximalen Nutzens verrechnet werden dürfen; ${ }^{6}$ das veranlasst die Vertreter einer Eingriffsrechtfertigung wegen überwiegenden Interesses aber ebenso wenig zu einer Aufgabe ihres Eingriffsprinzips wie die Tatsache, dass die Existenz unantastbarer Freiheit, wie sie auch in der Verfassung vorausgesetzt wird, unter dem Verdikt der Nutzenmaximierung nicht zu erklären ist. ${ }^{7}$

Der Grundmangel ${ }^{8}$ dieser Abwägungslehren sitzt jedoch tiefer: Das Recht scheint bei ihnen dann auf den Plan zu treten, wenn zwei (oder mehrere) Personen unvermittelt mit ihren „Gütern“ bzw. „Interessen“ aufeinander treffen und kollidieren. Diese scheinbar in keinem Zusammenhang stehenden Positionen sollen dann irgendwie bewertet und verrechnet werden. Ein Verständnis des Rechtseingriffs, das dem Einen die „Interessen" des je Anderen oder der Allgemeinheit unverbunden gegenüberstellt und diese gegeneinander "abwägt“, erweist sich vor dem Hintergrund eines personal-freiheitlichen Rechtsverständnisses als nicht haltbar, wie nun zu zeigen sein wird.

\section{Das Verhältnis des Einen zum Anderen nach einem freiheitlichen Rechtsbegriff}

Bei dem oben beschriebenen Versuch der Rechtfertigung durch Interessenabwägung wird bereits im Ansatz übersehen, dass die Rechtspositionen aller Personen eine gemeinsame Basis haben, von der ausgehend die weiteren Fragen zu lösen sind: Die Freiheit als Ausgangspunkt der Rechtsbegründung. Um eine solche Rechtsbegründung hat sich im Anschluss vor allem an Kant, Hegel und Fichte eine (mittlerweile recht große) Gruppe von (Straf-)Rechtswissenschaftlern bemüht.9

6 Kühnbach, Solidaritätspflichten Unbeteiligter. Dargelegt am Beispiel von Aggressivnotstand, Defensivnotstand, unterlassener Hilfeleistung und polizeilichem Notstand, 2007, S. 49.

7 Renzikowski (Fn. 5), S. 205. Siehe auch die Kritik von Köhler, Strafrecht, Allgemeiner Teil, 1997, S. 283: Das „Rechtsprinzip duldet keinerlei Einsatz auch nur einer einzigen Rechtsperson als Gegenstand für andere, da sie ,Zweck an sich“ ist.“ Treffend Kant, Handschriftlicher Nachlass. Moralphilosophie, Rechtsphilosophie und Religionsphilosophie. Reflexionen zur Moralphilosophie 6586, Akademieausgabe, Band XIX, S. 97: „Der Nutzen vieler gibt ihnen kein Recht gegen einen.“

8 Siehe zu weiteren Problemen der Abwägungslehren noch unten (III.) bei der Konkretisierung der einzelnen Eingriffsvoraussetzungen nach hiesigem Verständnis.

9 Grundlegend E.A.Wolff, ZStW 97 (1985), 786 ff.; ders., in: Hassemer (Hrsg.), Strafrechtspolitik, 1987, S. 137 ff.; Köhler, Der Begriff der Strafe, 1986; ders. (Fn. 7); ders., 


\section{Selbstbestimmung und intersubjektive Willkürkoordination}

Mit der Freiheit des Subjekts, die das Fundament des Rechts ist, ${ }^{10}$ ist gemeint zum einen die Möglichkeit der Emanzipation der inneren Willenssetzung von Natursachverhalten, d.h. von empirischen Vorgaben, zum anderen - und darauf aufbauend - die Freiheit, nach eigenen, selbst gewählten Zwecken zu handeln. ${ }^{11}$ Der Mensch handelt aufgrund seiner Vernunft nach selbstgesetzten Maximen, ist damit trotz seines Eingelassenseins in die äußere Welt und die Naturbedingungen selbst-, nicht fremdbestimmt. ${ }^{12}$ Die so beschriebene Autonomie ist ein "Grundsachverhalt" menschlichen Daseins. ${ }^{13}$

Allerdings kann sich der Mensch nicht als isoliertes Einzelwesen dieser Beziehung zwischen Natur und Selbstbestimmung bewusst werden. ${ }^{14} \mathrm{Da}-$ mit das Subjekt seine Beziehung zur umgebenden Welt begreifen und sich

Recht und Gerechtigkeit, 2017; Kablo, Die Handlungsform der Unterlassung als Kriminaldelikt, 2001; Zaczyk, Das Strafrecht in der Rechtslehre J. G. Fichtes, 1981; ders., Das Unrecht der versuchten Tat, 1989; ders., Selbstsein und Recht, 2014. Darauf aufbauend etwa Gierhake, Begründung des Völkerstrafrechts auf der Grundlage der Kantischen Rechtslehre, 2005; dies., Der Zusammenhang von Freiheit, Sicherheit und Strafe im Recht, 2013; Harzer, Die tatbestandsmäßige Situation der unterlassenen Hilfeleistung gemäß $₫ 323$ c StGB, 1999; Kelker, Zur Legitimität von Gesinnungsmerkmalen im Strafrecht, 2007; Klesczewski, Die Rolle der Strafe in Hegels Theorie der bürgerlichen Gesellschaft, 1991; Murmann, Die Selbstverantwortung des Opfers im Strafrecht, 2005; Noltenius, Kriterien der Abgrenzung von Anstiftung und mittelbarer Täterschaft, 2003; dies., Die Europäische Idee der Freiheit und die Etablierung eines Europäischen Strafrechts, 2017; Rath, Das subjektive Rechtfertigungselement, 2002; Rösinger (Fn. 3); Stübinger, Das „idealisierte“ Strafrecht, 2008; von Freier, Recht und Pflicht in der medizinischen Humanforschung, 2009; Zabel, Schuldtypisierung als Begriffsanalyse, 2007.

10 Eindringlich Gierhake, Begründung des Völkerstrafrechts auf der Grundlage der Kantischen Rechtslehre, 2005, S. 35 ff.

11 Dazu Kant, Grundlegung zur Metaphysik der Sitten, BA 37 (hier jeweils zitiert nach der Werksausgabe von Weischedel, Band VII): „Ein jedes Ding der Natur wirkt nach Gesetzen. Nur ein vernünftiges Wesen hat das Vermögen, nach der Vorstellung der Gesetze, d.i. nach Prinzipien, zu handeln, oder einen Willen.“ Siehe dazu Gierhake (Fn. 10), S. 35 ff., insb. 36.

12 Gierhake (Fn. 10), S. 37. Siehe auch Murmann, in: Koriath u.a. (Hrsg.), Grundfragen des Strafrechts, 2010, S. 189 (196), Fn. 52: „Gemeint ist hier Freiheit im Sinne einer Selbstdetermination, also im Sinne der Fähigkeit der Person, sich nach eigenen Gründen zu entscheiden."

13 Zaczyk, Das Unrecht der versuchten Tat, 1989, S. 165.

14 Ebenso Gierhake (Fn. 10), S. 49, dort insb. Fn. 96; E.A.Wolff, ZStW 97 (1985), 786 (813). 
selbst gedanklich als endliches Wesen in die äußere Welt einordnen kann, ${ }^{15}$ damit es sich selbst als Grund praktischer Urteile erkennen kann, die seine äußeren Handlungen bestimmen, ${ }^{16}$ bedarf es eines Anstoßes: Identitätsbildung und Selbstwerdung sind nicht möglich ohne ein Gegenüber, ohne andere Menschen. ${ }^{17}$ Dies sieht man in der menschlichen Entwicklung ganz deutlich am Eltern-Kind-Verhältnis: Fichte begriff in diesem Zusammenhang die Erziehung des Kindes durch die Eltern treffend als „Aufforderung zur freien Selbsttätigkeit“. ${ }^{18,} 19$ Im Rückstoß, in der folgenden tätigen Anerkennung des Auffordernden als Vernünftigen durch den Aufgeforderten, erweist sich auch letzterer als Vernünftiger. ${ }^{20}$ Vernunft kann also nur Dasein haben in einem fortwährend praktizierten Verhältnis gegenseitiger Anerkennung. ${ }^{21}$

Die Einsicht in die Existenz und Beschaffenheit des je fremden Selbstbewusstseins, ${ }^{22}$ das Erkennen des je Anderen als eines ebenfalls Vernünfti-

15 Vgl. Zaczyk, in: Kahlo/Wolff/Zaczyk (Hrsg.), Fichtes Lehre vom Rechtsverhältnis, 1992, S. 9 (15 f.).

16 Zaczyk, in: FS Wolff, 1998, S. 509 (517); ders., Strafrechtliches Unrecht und die Selbstverantwortung des Verletzten, 1993, S. 21.

17 Die hier aufzuzeigende Bedeutung der Sozialität der Menschen wird im Fortgang der Überlegungen zum Notrecht entscheidend sein.

18 Fichte, Grundlage des Naturrechts, $\$ 3$ (Corollaria), S. 39 (zitiert nach der Ausgabe von I.H. Fichte, Band III, 1971); dazu Zaczyk, in: Hoffmann (Hrsg.), Das Recht als Form der „Gemeinschaft freier Wesen als solcher“, 2014, S. 25 (32); ders., in: GS Heinze, 2005, S. 1111 (1118f.); ders. (Fn. 13), S. 158 mit dem Hinweis, dass die Aufforderung von Fichte „nicht nur als genetisch (zur Entstehung des Selbstbewusstseins) notwendig eingeführt, sondern als mit ihm - gleichsam zeitlebens notwendig verknüpft" wird.

19 Die Bedeutung der Interpersonalität für den Bereich praktischer Handlungen ist auch in Kants kategorischen Imperativ angelegt, s. dazu Gierhake (Fn. 10), S. 41 und noch sogleich im Text. In Fichtes Rechtsphilosophie ist Interpersonalität dagegen nicht erst auf Ebene praktischer Handlungen, sondern schon als Bedingung des Selbstbewusstseins relevant, dazu Zaczyk, in: Hoffmann (Hrsg.) (Fn. 18), S. 25 $(30,32)$.

20 Diesen Gedankengang bei Fichte (Fn. 18), S. 47 erläutert Zaczyk (Fn. 13), S. 161. Autonomie setzt damit immer auch den Anderen voraus (s. dazu Zaczyk [Fn. 13], S. 165).

21 Fichte bestimmt eben dieses Verhältnis als das Rechtsverhältnis, s. Grundlage des Naturrechts (Fn. 18), S. 41. Dazu Zaczyk (Fn. 13), S. 161.

22 Dazu Zaczyk, in: FS Wolff, 1998, S. 509 (517); vertiefend ders., in: Hoffmann (Hrsg.) (Fn. 18), S. 25 (32); ders., in: GS Heinze (Fn. 18), S. 1111 ff., insb. 1118 f. in Auseinandersetzung mit Fichtes „Begründung der Personalität aus Interpersonalität“ (S. 1115). Siehe dazu und zum Folgenden auch Gierhake (Fn. 10), S. 49. 
gen, hat nun wesentliche Folgen für die Bestimmung des richtigen Umgangs miteinander:

Von diesem Ausgangspunkt der intersubjektiven Bestimmung von Subjektivität wird deutlich, dass jedem Einzelnen ein selbstbestimmtes Leben möglich sein muss. Die dadurch bedingte Notwendigkeit einer Verallgemeinerungsleistung ist bereits in Kants kategorischem Imperativ angelegt: Handlungsleitendes Prinzip kann nur ein solches sein, das den anderen in seiner Selbstbestimmung mit einbezieht. Ein allgemeines Moralprinzip schließt es aus, individuelle Nutzenmaximierung oder Glücksempfinden zum Maßstab guten Handelns zu machen. ${ }^{23}$ Nach Kant ist ein Handeln daher nur dann gut, wenn die zugrundeliegende Maxime widerspruchslos verallgemeinert werden kann: „Handle nur nach derjenigen Maxime, durch die du zugleich wollen kannst, dass sie ein allgemeines Gesetz werde. " 24

Allein dadurch, dass ein jeder (sei es nach bestem Wissen und Gewissen) nach aus seiner Sicht verallgemeinerbaren Maximen handelt, ist ein freiheitliches Miteinander aber noch nicht gesichert. Denn die zu erbringende Leistung der Anerkennung ist Schwächen ausgesetzt, ${ }^{25}$ ist der Mensch doch nicht nur Vernunftwesen, sondern auch naturhaftes Ich, ${ }^{26}$ das mit den eigenen Trieben und der eigenen Endlichkeit konfrontiert ist. Es kann unter den Beteiligten nicht nur deswegen zu Willenswidersprüchen kommen, weil sich einer von ihnen bewusst in Widerspruch zum Richtigen setzt, sondern auch, weil er schlicht die Sachlage verkennt, sich irrt oder sich etwas vormacht. ${ }^{27}$ Das Ich kann sich aber in eigener Vernunftleistung auf die Schliche kommen: ${ }^{28}$ Es erfasst sowohl „sich selbst in seiner Wechselwirkung zwischen Naturhaftigkeit und begründender Vernunft" ${ }^{29}$ als auch den Anderen, und bezieht ihn in diese Objektivierung mit ein. Es gilt, eine als gemeinsam erkannte äußere Seite des Daseins zu meistern. ${ }^{30}$ Diese notwendig gemeinsame Äußerlichkeit durch die Begeg-

23 Siehe dazu Gierhake (Fn. 10), S. 39 f.

24 Kant, Grundlegung zur Metaphysik der Sitten, BA 52.

25 Zaczyk, Strafrechtliches Unrecht und die Selbstverantwortung des Verletzten, 1993, S. 24.

26 E.A.Wolff, ZStW 97 (1985), 786 (812); dazu auch Gierhake (Fn. 10), S. 49 f.

27 Siehe dazu E.A.Wolff, ZStW 97 (1985), 786 (814 f.); Gierhake (Fn. 10), S. 49.

28 Diese schöne Formulierung findet sich bei E.A.Wolff, ZStW 97 (1985), 786 (813).

29 So E.A.Wolff, ZStW 97 (1985), 786 (813) zur vom selbstbewussten Einzelnen vollzogenen Verobjektivierung der Mängel der eigenen Denkleistung; zur entsprechenden Methode beim Einbezug des Anderen dort S. 815.

30 Vgl. E.A.Wolff, ZStW 97 (1985), 786 (815); ders., in: Hassemer (Hrsg.) (Fn. 9), S. 137 (140). 
nung mehrerer Menschen auf der einen Erde wird von Kant an verschiedenen Stellen mit dem Verweis auf deren Kugelgestalt anschaulich in Bezug genommen. ${ }^{31}$

Der Einzelne allein kann also auch mithilfe des kategorischen Imperativs nicht allgemeinverbindliche Regeln für den je Anderen aufstellen; ${ }^{32}$ es bedarf vielmehr einer gemeinschaftlichen Leistung, die das Vermögen des Einzelnen, der stets in seiner subjektiven Perspektive verhaftet ist, übersteigt. ${ }^{33}$ Diese erforderliche gemeinschaftliche Vernunftleistung ist das Recht: ${ }^{34}$ Die Menschen, die sich wechselseitig sowohl als Vernunftwesen, als auch in ihrem Eingebundensein in die Endlichkeit auf der einen Erde anerkennen, beschränken ihre eigenen Handlungsmöglichkeiten durch den Begriff der Freiheit der anderen: ${ }^{35}$ Recht ist „der Inbegriff der Bedingungen, unter denen die Willkür des Einen mit der Willkür des Anderen nach einem allgemeinen Gesetze der Freiheit zusammen vereinigt werden kann“. ${ }^{36}$ Das Recht regelt folglich - in Abgrenzung zur Moral ${ }^{37}$ - die $\ddot{a} u ß e-$ $r e$ Beziehung freier Vernunftsubjekte zueinander. ${ }^{38}$ Hierbei fragt es nach der Vereinbarkeit der äußeren Handlung des Einen mit der Freiheit des Anderen nach Maßgabe eines allgemeinen Gesetzes und damit - analog

31 Kant, Metaphysik der Sitten, Rechtslehre, $\ 13$, AB 84, 85; $\mathbb{4} 43$, A 161, 162/B 191, 192; $\mathbb{S} 62$, A 229/B 259 (hier jeweils zitiert nach der Werksausgabe von Weischedel, Band VIII). S. dazu Zaczyk, in: Gephart/Suntrup (Hrsg.), Rechtsanalyse als Kulturforschung, Bd. II, 2015, S. 19 (24); s. auch E.A.Wolff, ZStW 97 (1985), 786 (815).

32 Gierhake (Fn. 10), S. 52. Ausführlich Zaczyk (Fn. 13), S. $146 \mathrm{ff}$.

33 Siehe dazu, dass die Individuen „einer ihre individuelle Perspektive übersteigenden Gestaltungsobjektivität“ bedürfen, „um das friedliche Zusammenleben zu organisieren“ Gierhake (Fn. 10), S. 54. Siehe zu diesem „perspektivischen Moment" auch E.A.Wolff, ZStW 97 (1985), 786 (815).

34 Siehe Gierhake (Fn. 10), S. 70.

35 Vgl. Murmann (Fn. 9), S. 196, siehe dort auch S. 163: „Die wechselseitige Einsicht in die Vernünftigkeit des je Anderen ist die Basis für ein Verhältnis der Gleichheit der Beteiligten im Hinblick auf die Vernunft und die Grundlage eines gegenseitigen Anerkennungsverhältnisses, das die rechtliche Beziehung kennzeichnet“.

36 Kant, Metaphysik der Sitten, Einleitung in die Rechtslehre, $\mathbb{B}$, AB 33. Siehe dazu Zaczyk (Fn. 13), S. $151 \mathrm{f}$.

37 Siehe zur Unterscheidung von Moralität und Legalität Zaczyk, in: Gephart/ Suntrup (Hrsg.) (Fn. 31), S. 19 (28 f.) sowie ders., JRE 14 (2006), 311 ff.; Gierhake (Fn. 10), S. $56 \mathrm{ff}$.

38 Kant, Metaphysik der Sitten, Einleitung in die Rechtslehre, $\mathbb{S}$ B, AB 32, 33: Der Begriff des Rechts betrifft „nur das äußere und zwar das praktische Verhältnis einer Person gegen eine andere, sofern ihre Handlungen als Facta aufeinander (unmittelbar, oder mittelbar) Einfluss haben können“. S. dazu auch Gierhake (Fn. 10), S. 54; Zaczyk, in: Gephart/Suntrup (Hrsg.) (Fn. 31), S. 19 (25 f.). 
zum kategorischen Imperativ - nach einer insoweit formalen Vereinbarkeit der Handlungen, ohne inhaltliche Anforderungen insbesondere hinsichtlich eines mit den Handlungen zu verfolgenden Zwecks zu stellen. ${ }^{39}$ Jeder kann seine Freiheitssphäre, d.h. den Raum der eigenen Willkür, ausfüllen, wie er will, solange sich dies mit der Selbstbestimmung des Anderen verträgt. ${ }^{40}$ Geht es um die Koordination der Willkür des Einen mit der des Anderen, so muss der Handelnde auch lediglich auf eben diese Willkür des Anderen, nicht auf dessen Wünsche Rücksicht nehmen, ${ }^{41}$ denn „der Handelnde verlöre seinen äußeren Selbst-Stand, müsste er sein Handeln an der Innerlichkeit des Anderen orientieren". ${ }^{42}$

\section{Erste Form der Eingriffsrechtfertigung: Zwangsbefugnis nach dem Satz vom Widerspruch bei Verstoß gegen das Verletzungsverbot}

Unmittelbar einsichtig ist bei Zugrundelegung des soeben entfalteten Rechtsbegriffs eine erste Form der Eingriffsrechtfertigung, die sich aus der Abgrenzung von Räumen eigener Willkür ergibt: Überschreitet eine Person ihre Freiheitssphäre und dringt gleichsam in die Sphäre einer anderen ein, so darf letztere den Übergriff zurückweisen. Ihr kommt mit Kant eine Zwangsbefugnis nach dem Satz vom Widerspruch zu:

"(W)enn ein gewisser Gebrauch der Freiheit selbst ein Hindernis der Freiheit nach allgemeinen Gesetzen (d.i. unrecht) ist, so ist der Zwang, der diesem entgegengesetzt wird, als Verhinderung eines Hindernisses der Freiheit mit der Freiheit nach allgemeinen Gesetzen zusammenstimmend,

39 Kant, Metaphysik der Sitten, Einleitung in die Rechtslehre, $\mathbb{~ B , ~ A ~ 3 3 / B ~ 3 3 , ~} 34$ sowie in der Tugendlehre A 7. Siehe zum Ganzen auch Gierhake (Fn. 10), S. 60 f. mit Fn. 134 in Abgrenzung zu Rechtsauffassungen in der Tradition der utilitaristischen Ethik, wonach sich die Richtigkeit einer Handlung nach der Nützlichkeit ihrer Folgen beurteilt. S. dort auch S. 37 ff. zur auf das jeweilige Subjekt beschränkten Gültigkeit von auf Zweckerreichung gerichteten Sollensnormen bei Kant.

40 Zaczyk, in: Gephart/Suntrup (Hrsg.) (Fn. 31), S. 19 (26). Dass das Recht jedoch unvollständig begriffen wäre, wenn man als dessen Ziel lediglich die Separierung der Einzelnen voneinander ansehen würde, wird unten bei Punkt 3. noch genauer zu zeigen sein.

41 S. Kant, Metaphysik der Sitten, Einleitung in die Rechtslehre, $₫$ B, AB 32, 33.

42 Zaczyk, in: Gephart/Suntrup (Hrsg.) (Fn. 31), S. 19 (26). 
d.i. recht; mithin ist mit dem Rechte zugleich eine Befugnis, den, der ihm Abbruch tut, zu zwingen, nach dem Satze des Widerspruchs verknüpft." ${ }^{3}$

Ein Eingriff ist also wegen Unrechtsverantwortung des Eingriffsadressaten gerechtfertigt. Um eine solche „Rechtfertigung mittels bestimmter $\mathrm{Zu}$ rechnungsprinzipien" 44 handelt es sich vor allem bei der Notwehr $(\mathbb{\Omega} 32$ StGB) sowie entsprechend auf institutioneller Ebene beim polizeilichen Vorgehen des Handlungsstörers, aber auch beim sog. Defensivnotstand $(\mathbb{} 228 \mathrm{BGB})$ und der polizeilichen Abwendung einer Zustandsstörung. ${ }^{45}$

Ein derartiger Anknüpfungspunkt der (zumindest objektiven) Zurechnung der Gefahr fehlt allerdings beim Eingriffsadressaten des sog. Aggressivnotstandes. Warum darf man auf ihn zugreifen, auch wenn er sich mit seinen Handlungen in seiner Freiheitssphäre hält?

Damit der Eingriff im Aggressivnotstand nicht Instrumentalisierung ist, muss sein Grund auch hier in der Person des Betroffenen gefunden werden.

\section{Sozialität und Freiheitsermöglichung}

Wäre das Recht mit der (negativen) Abgrenzung der Freiheitssphären bereits vollständig beschrieben, ginge es um ein bestmögliches Nebeneinander freier Einzelner, die sich gegenseitig - sobald sie sich einmal in Gemeinschaft Regeln gegeben haben - nicht in die Quere kommen. Es gibt aber noch einen weitergehenden, (positiven) Freiheitsaspekt, der aus dem bisher Gesagten folgt: Der Mensch als der Ausgangspunkt, von dem aus Recht einzig sinnvoll gedacht werden kann, ist durch Freiheit und Soziali-

43 Kant, Metaphysik der Sitten, Einleitung in die Rechtslehre, $\sqrt{ } \mathrm{D}, \mathrm{AB} 35$. Siehe dazu Zaczyk (Fn. 13), S. 154; Gierhake (Fn. 10), S. 62 ff.

44 Frister, Schuldprinzip, Verbot der Verdachtsstrafe und Unschuldsvermutung als materielle Grundprinzipien des Strafrechts, 1988, S.30; s. dazu auch Rösinger (Fn. 3), S. $171 \mathrm{f}$.

45 Siehe dazu den Überblick bei Köhler, Recht und Gerechtigkeit, 2017, S. 286 ff.; grundlegend ders. (Fn. 7), S. 260 ff., 278 ff. Neumann, in: von Hirsch u. a. (Hrsg.), Solidarität im Strafrecht, 2013, S. 155 (167) spricht im Zusammenhang mit $\mathbb{} 228$ BGB und dem defensiven Notstand im Strafrecht von dem „Prinzip der besonderen Verantwortlichkeit für die eigene Sphäre“. Siehe auch weitergehend zu den Unterschieden zwischen Defensivnotstand („schwacher Verantwortung“) und Notwehr („starker Verantwortung“) Wilenmann, Freiheitsdistribution und Verantwortungsbegriff. Die Dogmatik des Defensivnotstands im Strafrecht, 2014, S. 457 ff., insb. S. 459. 
tät geprägt. Damit sind beide Elemente immer schon integrale Bestandteile des Begriffs des Rechts. ${ }^{46}$

Auch wenn das Recht Räume äußerer Handlungen (Willkür) koordiniert und nicht individuelle Wünsche ${ }^{47}$ sodass der Einzelne vor Eingriffen grundsätzlich dadurch geschützt ist, dass er sich in seiner eigenen Willkürsphäre hält und den je Anderen nicht in seiner Willkürausübung stört, so ist es deswegen trotzdem noch nicht unmöglich, die Not des je Anderen als Element einer Eingriffsrechtfertigung zu betrachten, die den Rückbezug auf die Person des Eingriffsadressaten gewährleistet. Ausgeschlossen ist es zwar bei Forderung eines solchen Rückbezugs, Eingriffe unmittelbar auf das empirisch-materiale Wobl eines anderen zu stützen. ${ }^{48}$ Dennoch kann ein Erhaltungsgebot in Not in gewissem Umfang als Rechtspflicht begründet werden, wenn man die Notwendigkeit logisch vorrangiger Freiheitsgewährung, die noch vor freiheitsschützenden Verboten steht, in den Blick nimmt. Was hiermit gemeint ist, wird deutlich, wenn der reale Weltbezug des Einzelnen in der Rechtsbegründung berücksichtigt wird: Tatsächlich ist in der einen Welt das endliche Wesen darauf angewiesen, seine Sphäre gegenüber der des notwendig mit ihm durch die Kugelgestalt ${ }^{49}$ verbundenen anderen endlichen Wesens abzugrenzen, damit jeder in Freiheit leben kann. An dieser Stelle wurde im Gedankengang ${ }^{50}$ deutlich, dass die Verbundenheit der Menschen durch die Geschlossenheit der Erdoberfläche als empirische Überlegung Eingang in die Rechtsbegründung finden muss. Denn der Mensch ist (ebenso wie er vernunftbegabt und Grund praktischer Urteile ist) in eine Wirklichkeit eingelassen, in der er auch betrachtet werden muss.

Wenn Selbstbestimmung also in der Realität betrachtet wird, besteht die Notwendigkeit, die (praktischen) Voraussetzungen von Selbstbestimmung umfassend in der Rechtsbegründung mitzuführen. Als wesentlich erkannt wurde insoweit die Beziehung zum je Anderen - die Sozialität als Voraussetzung von Selbstbewusstsein und der Personalität überhaupt, ${ }^{51}$

46 Ausführlich Zaczyk, in: GS Heinze (Fn. 18), S. 1111 (1114); dazu auch Rösinger, in: Gertz u. a. (Hrsg.), Diversity and Inclusion in Higher Education and Societal Contexts. International and Interdisciplinary Approaches, 2018, S. 237 ff.

47 Siehe bereits oben unter 1.

48 So in Kritik an den Abwägungslehren Köhler (Fn. 45), S. 263.

49 Siehe dazu bereits oben bei Fn. 31. Dieses Faktum wird uns durch die gegenwärtige Pandemie-Krise eindrücklich vor Augen geführt.

50 Siehe oben unter 1.

51 Insoweit wurde bereits oben (bei Fn. 18 ff.) unter Verweis auf Fichte die Sozialität als notwendig mit der Autonomie des Einzelnen verbunden eingeführt. 
letztlich als Voraussetzung der (für den Rechtsbegriff so zentralen) Selbstbestimmung. ${ }^{52}$ Aus diesem Zusammenhang erschließt sich die Bedeutung der Ursprungsgemeinsamkeit ${ }^{53}$ der Menschen für das Recht: Die im Begründungsgang des Rechts zentrale Forderung, dass Selbstbestimmung für alle möglich sein und daher eine entsprechende Verallgemeinerungsleistung erbracht werden muss, führt nicht nur zu dem Verbot, andere in ihrer Freiheit zu verletzen; diesem Verbot liegt vielmehr normlogisch und faktisch das Gebot der Freiheitsermöglichung voraus. ${ }^{54}$

Versteht man das Rechtsverhältnis als ein gegenseitiges Anerkennungsverhältnis, ${ }^{55}$ so dient das Recht also nicht etwa nur der trennenden Abgrenzung der Willenssphären: ${ }^{56}$ Steht der Mensch in seiner Vernunftexistenz in einem notwendigen Zusammenhang mit anderen, so folgt daraus eine Grundgleichheit in der fundamentalen Rechtsfähigkeit, mit der ein Anspruch auf die erforderliche materiale Grundteilhabe verbunden ist. ${ }^{57}$

52 Siehe zum Ganzen auch Zaczyk, in: FS Wolff (Fn. 22), S. 509 (519): „An dieser Stelle wird klar, wie aus dieser Einsicht in die Beschaffenheit ,bloßer 'Vernunft der Rechtsgedanke sich entwickelt und entwickeln muss. Freiheit ist nicht nur je meine, sondern gleichursprünglich die des Anderen, dem Recht immanent ist damit Nähe und Distanz zu anderen Personen gleichermaßen.“

53 Dazu auch Köhler (Fn. 45), S. 289. Siehe dazu, dass „das Subjekt als solches notwendig als in einem Zusammenhang mit anderen stehend begriffen werden muss“, auch Zaczyk, in: FS Wolff (Fn. 22), S. 509 (519).

54 So Köhler, in: Klesczewski u.a. (Hrsg.), Kants Lehre vom richtigen Recht, 2005, S. 123 (128); ders. (Fn. 45), S. 262, 265. Dazu auch E.A. Wolff, ZStW 97 (1985), 786 (815); Gierhake (Fn. 10), S. 51. Köhler (Fn. 7), S. 208 verweist auf das „normative Vernunftgebot, selbstzweckhaft zu existieren“, in dessen Folge nicht nur äußere Freiheitssphären definiert und entsprechende Verbotsnormen aufgestellt werden; vielmehr müssten auch die Daseinsbedingungen i.S. positiver Freiheit miteinbezogen werden.

55 Ausführliche Begründung dieses Zusammenhangs bei E.A.Wolff, in: Hassemer (Hrsg.) (Fn.9), S.137 (162 ff.); Zaczyk (Fn.13), S. 128 ff. Siehe auch Zaczyk (Fn. 25), S. 22 zum äußeren Interpersonalverhältnis als „Basis“ des Rechts.

56 E.A.Wolff, ZStW 97 (1985), 786 (815).

57 Von Freier (Fn. 9), S. 172; s. auch dort S. 173: „Auf dieser Einsicht in unabdingbare Materialisierungen beruht die Hegelsche Theorie des Notrechts". Siehe aber gegen die Hegel'schen Materialisierungen Helmers (Fn. 2), S. $183 \mathrm{ff}$. 


\section{Zweite Form der Eingriffsrechtfertigung: Begrenzte Solidaritätspflicht zur Aufrechterhaltung des Rechtsverhältnisses}

Was folgt aus dem Gesagten für die Eingriffsrechtfertigung gegenüber einer Person, die für die Gefahr nicht verantwortlich ist? Ausgeschlossen ist nach dem zugrunde gelegten Rechtsbegriff jedenfalls ein umfassendes Notrecht: Der Begriff der (äußeren) Freiheit selbst impliziert, dass jeder die empirische Bedingtheit seiner Freiheitssphären mit ihren Zufälligkeiten zu tragen hat. ${ }^{58}$ Es gilt der Grundsatz casum sentit dominus. ${ }^{59}$ Gerät eine Person in Not, ist es in einem Rechtsverhältnis freier Personen ausgeschlossen, die zufällige Gefahr auf einen zufällig begegnenden Anderen einfach zu verlagern. Das könnte keine interpersonal gültige Regel sein. ${ }^{60}$

Möglich ist aber ein begrenztes Notrecht, das auf dem menschenrechtlichen Grundverhältnis ${ }^{61}$ der wechselseitigen Ermöglichung und Achtung der Freiheit beruht: Nicht nur muss im Verhältnis der Familie zum noch abhängigen Kind dieses zunächst in seinen Fähigkeiten gebildet werden; ${ }^{62}$ auch wenn die Menschen bereits einen grundsätzlich gefestigten Selbststand erreicht haben, aktualisiert sich doch in der zufälligen existentiellen Not eben dieses Grundverhältnis, und zwar in einem Solidaritätsgebot: Die Grundbedingungen eines Lebens in Freiheit müssen für jeden Einzelnen erhalten bleiben. Sind die substantiellen Teilhabebedingungen des Einen in Gefahr (vor allem durch den eigenen Tod, aber auch durch erhebliche Körperverletzungen) und lässt sich die Gefahr nicht anders abwenden als durch den (in seinen Grenzen noch näher zu bestimmenden)

58 Siehe Köhler (Fn. 7), S. 282; ders. (Fn. 45), S. 289.

59 Köhler (Fn. 45), S. 289.

60 So Köhler (Fn. 7), S. 282. Insoweit berechtigt ist auch die Ablehnung eines allgemeinen Notrechts bei Kant, s. Kant, Metaphysik der Sitten, Anhang zur Einleitung in die Rechtslehre, AB 41, 42, S. 343 f. Siehe auch dazu, dass die häufig suggerierte weitergehende Ablehnung jeglichen Notrechts durch Kant bei Betrachtung seines Gesamtwerks nicht unzweifelhaft ist, Helmers (Fn. 2), S. 137 ff., insb. $142 \mathrm{ff}$. mit einer Auswertung der Literatur.

61 Köbler (Fn. 7), S. 280 ff.; von Freier (Fn. 9), S. 155 ff.; Helmers (Fn. 2), S. 292 ff. Siehe auch den Überblick bei Köhler (Fn. 45), S. 288 ff. sowie zum Grundgedanken dort S. 277 ff. Köhler entwickelt das genannte menschenrechtliche Grundverhältnis für den Notstand allerdings vor allem als besitzrechtliches Grundverhältnis weiter, so ausdrücklich in Recht und Gerechtigkeit (Fn. 45), S. 289; ebenso Helmers (Fn. 2), S. 248 ff. Siehe zu den hieraus gezogenen Konsequenzen für die Eingriffsvoraussetzungen des Notstands noch genauer unten III. 2) a). Davon abweichend wird hier im Folgenden das Notrecht ohne Beschränkung auf die besitzrechtliche Perspektive stärker mit dem Anerkennungsverhältnis selbst verbunden.

62 Siehe zur familiären Personensorgepflicht Köhler (Fn. 54), S. 123 (129). 
Eingriff in eine grundsätzlich dem Anderen zugeordnete Rechtsposition, so kann sich letzterer nicht auf diese Position berufen, wenn durch das Beharren auf den Status quo die Existenz des Gefährdeten und damit die Grundlage des rechtlichen Verhältnisses selbst aufgehoben würde. ${ }^{63}$

Die Grundteilhabe wird hier unmittelbar als Forderung des Anerkennungsverhältnisses verstanden: Bei der Gefahr des Ausscheidens des Einen aus dem Rechtsverhältnis und damit des Erlöschens des interpersonalen Rechtsverhältnisses kann der Andere sich nicht von Rechts wegen auf das ihm sonst Zustehende berufen, wenn er selbst durch den Eingriff lediglich partiell-ersetzlich betroffen wäre. ${ }^{64}$ In der Konstellation des Aggressivnotstandes sind die Berufung des Eingriffsadressaten auf das Verletzungsverbot und damit seine Rechtsbehauptung widersprüchlich, „wenn sie die Anerkennung von Rechten in einem Verhältnis verlangen, das dadurch gegen den Willen des Adressaten seinem Untergang preisgegeben wird“. ${ }^{65}$ Die Pflicht des Eingriffsadressaten im Notstand beruht auf dem Gebot, die

63 In diesem Sinne auch von Freier (Fn.9); ähnlich Köhler (Fn. 45), S. 289 unten: "Grundwiderspruch zum Recht der Menschheit in allen Subjekten“; siehe auch bereits ders., ZStW 107 (1995), 10 (15, 20) zur Rechtfertigung des Freiheitseingriffs im Notstand „aus dem gemeinsam vorausgesetzten Grunde, das existierende Rechtsverhältnis in einem seiner Mitkonstituenten zu erhalten“.

64 Siehe dazu insb. die Ausführungen Hegels zum Notrecht in den Grundlinien der Philosophie des Rechts, $\mathbb{} 127$ (zitiert nach der Ausgabe Georg Wilhelm Friedrich Hegel: Werke. Auf der Grundlage der Werke von 1832-1845 neu edierte Ausgabe. Redaktion Moldenhauer/Michel, Band 7, 1986.): „Die Besonderheit der Interessen des natürlichen Willens, in die einfache Totalität zusammengefasst, ist das persönliche Dasein als Leben. Dieses in der letzten Gefahr und in der Kollision mit dem rechtlichen Eigentum eines anderen hat ein Notrecht (nicht als Billigkeit, sondern als Recht) anzusprechen, indem auf der einen Seite die unendliche Verletzung des Daseins und darin die totale Rechtlosigkeit, auf der anderen Seite nur die Verletzung eines einzelnen beschränkten Daseins der Freiheit steht, wobei zugleich das Recht als solches und die Rechtsfähigkeit des nur in diesem Eigentum Verletzten anerkannt wird.“ Siehe zur Begrenzung des Noteingriffs auf „partikulär-ersetzliche“ Rechtsgüter Köhler (Fn. 7), S. 281, 290; ders., ZStW 107 (1995), 10 (20) sowie unten III.

65 So von Freier (Fn. 9), S. 173 im Anschluss an Hegels Konzeption. Siehe auch dort S. 177: „Der Eigentümer beruft sich widersprüchlicherweise auf das abstrakte Rechtsverhältnis, da das konkrete Verhältnis damit seine Aufhebung impliziert. Das Rechtsverhältnis verkehrt sich zur Rechtlosstellung." Siehe zudem Köhler (Fn. 45), S. 289, Fn. 198, dazu, dass das von Hegel angenommene Notrecht in der Kantischen Soziallehre (gemeint ist im Gedanken des ursprünglichen Gesamtbesitzes) bereits angelegt sei. Dagegen meint Kühnbach (Fn. 6), S. 109 ff., dass die Kantische Freiheitskonzeption wegen ihrer Formalität ungeeignet sei, den Realitätsbezug von Freiheit aufzunehmen. 
Vernunftexistenz des je Anderen und damit letztlich das Rechtsverhältnis selbst gegen das „vernunftlose Walten des Zufalls“66 ${ }^{\text {zu erhalten. }}{ }^{67}$

Dem kategorischen Grund genügen gerade nicht bloß pragmatische Erwägungen bzgl. eines wechselseitigen Bedürfnisses nach Hilfe („kontraktualistisch-utilitaristisches Kalkül“). ${ }^{68}$ Die Erwägung, man solle helfen, damit einem in ähnlicher Situation selbst geholfen werde, genügt für eine allgemeingültige Regel nicht; „denn sie hinge ab von der Wohleinschätzung des einzelnen, ob sie künftig überhaupt selbst noch Hilfe brauchten und wollten, ob nicht die Härte des Lebens vorzuziehen sei."69 Nicht ein Kalkül, sondern die aus dem Rechtsbegriff selbst folgende Grundsolidaritätspflicht aller ${ }^{70}$ aktualisiert sich in der Situation existentieller Not. ${ }^{71}$ Der Grund für das Notrecht ebenso wie der für die allgemeine Hilfeleistungs-

66 So Köhler (Fn. 45), S. 670.

67 Siehe dazu auch Köhler (Fn. 7), S. 208: Der äußere Zufall sei dem an sich schlüssig entwickelten Dasein freier Subjekte zuwider. Daher entwickeln sie in wechselseitig-allgemeiner Anstrengung bspw. die ärztliche Heilkunst. „Die Pflicht zur Selbstaffirmation vernünftigen Daseins übersetzt sich in eine Pflicht, die äußere Zufallsausgesetztheit möglichst allgemein aufzuheben." Dazu auch E.A.Wolff, in: Hassemer (Hrsg.) (Fn. 9), S. 137 (177): Würde man nichts geben und nichts nehmen wollen, würde man sich ,in einer entscheidenden Stelle der Mechanik des Zufalls ausliefer(n) und einen Bereich, in dem vernünftige Beurteilung möglich wäre und der von der Vernunft gestaltet werden könnte, der Macht der Vernunft entzieh(en).“

68 Siehe dazu und zum Folgenden Köhler (Fn. 7), S. 207.

69 Ebendies ist auch gegen Ansätze einzuwenden, die auf den Rawls'schen Schleier des Nichtwissens abstellen, so etwa Merkel, in: Institut für Kriminalwissenschaften Frankfurt a.M. (Hrsg.), Vom unmöglichen Zustand des Strafrechts, 1995, S. 183 (185); Coninx, Das Solidaritätsprinzip im Lebensnotstand, 2012, S. 14, $21 \mathrm{ff} ., 101 \mathrm{ff}$. S. dazu die kritische Auseinandersetzung von Stübinger, NotwehrFolter und Notstands-Tötung? Studien zum Schutz von Würde und Leben durch Recht, Moral und Politik, 2015, S. 281 ff. sowie Pawlik, JRE 22 (2014), 137 (147 ff.).

70 Köbler (Fn. 7), S. 309. S. auch dens., in: Klesczewski u.a. (Hrsg.) (Fn. 54), S. 123 (126ff.) sowie Neumann, in: von Hirsch u.a. (Hrsg.) (Fn. 45), S. 155 (165) zur Begründung der Solidarität als Rechtspflicht (im Gegensatz zur bloßen moralischen Pflicht). Instruktiv zur Geschichte des Solidaritätsbegriffs Stübinger (Fn.69), S. $288 \mathrm{ff}$.

71 Durch diese Herleitung der Solidaritätspflicht trifft den hiesigen Ansatz nicht die Kritik, die vielerorts (berechtigt) gegen die Verwendung des Solidaritätsbegriffs im (straf-)rechtlichen Kontext erhoben wird, nämlich den legitimatorischen Mehrwert unausgewiesen zu lassen (so etwa Pawlik, JRE 22 [2014], 137 ff. [143]; siehe auch zusammenfassend zur vorgebrachten Kritik ders. [Fn. 2], S. 58 f.) und lediglich als leere Parole zu dienen („diffus“ zu bleiben, so Helmers [Fn. 2], S. 282, s. dort auch S. 327). 
pflicht liegt damit in der oben beschriebenen Ursprungsgemeinsamkeit ${ }^{72}$ der Menschen, die eine materiale Grundteilhabe für jeden Einzelnen verbürgt.

Neben die Inanspruchnahme wegen einer (Vor-)Verantwortlichkeit ${ }^{73}$ tritt also die Inanspruchnahme aus dem Gedanken der Teilhabe am Rechtsverhältnis, dem eine Pflicht zur Solidarität entspricht. Dieser Gedanke liegt auch weiteren Eingriffen im institutionellen Verhältnis zugrunde, etwa der polizeilichen Nichtstörerhaftung, der Enteignung, der Pflicht zur Sozialhilfe ${ }^{74}$ und auch dem Strafverfahrenseingriff. ${ }^{75}$ Die Eingriffsgründe des Notrechts und der allgemeinen Hilfspflicht, ${ }^{76}$ die schon im unmittelbaren, interpersonalen Verhältnis der zufälligen Begegnung gelten, werden in den jeweiligen institutionellen Zusammenhängen modifiziert. ${ }^{77}$ Jedoch bleibt das erarbeitete vorpositive Rechtfertigungsprinzip für das gesamte Rechtssystem prägend. ${ }^{78}$

Die bisherige Untersuchung hat damit gezeigt, dass der Eingriff des Einen in die Rechtspositionen des Anderen gegen dessen Willen zwei grundlegende und voneinander zu unterscheidende Formen des Rückbezugs auf die Person des Eingriffsadressaten kennt, die die Prüfung der Rechtmäßigkeit eines Eingriffs notwendig vorstrukturieren: Die Haftung aus dem Grund der Unrechtsverantwortung und die Teilhabehaftung.

72 So auch Köhler (Fn. 45), S. 289.

73 Dazu oben unter 2.

74 Dazu auch Köhler (Fn. 45), S. 670; ders. (Fn. 7), S. 208 f.; ders., in: Klesczewski u.a. (Hrsg.) (Fn. 54), S. 123 (137). Nach E.A.Wolff, ZStW 97 (1985), 786 (815) „liegt im gegenseitigen Anerkennungsverhältnis gegenüber dem Anderen auch die Pflicht, Behinderungen der Ausgangslage auszugleichen. Die sozialen Leistungen sind deswegen Teil des Begründungsverhältnisses“.

75 Siehe dazu Köhler, ZStW 107 (1995), 10 ff. sowie ausführlich Rösinger (Fn. 3).

76 Nach Köhler (Fn. 45), S. 284 ff. ist das vorgängige Gebot der Hilfeleistungspflicht bzw. Ermöglichungspflicht (dazu a.a.O. S. 260 ff.) außerhalb der Rechtfertigungsgründe für Eingriffe zu betrachten. Es handelt sich für ihn bei dieser Pflicht also nicht um einen Eingriff im engeren/technischen Sinn. Auch wenn danach die Rechtfertigungsgründe als „Befugnisse (Erlaubnisse) zum Eingriff in den primären grundrechtlichen Status" von dem Hilfsgebot zu trennen sind, verweist er darauf, dass diese Befugnisse ebenfalls „im allgemeinen Prinzip rechtgesetzlicher Selbstbestimmung“ gründen, „das dem primären Verbot/Gebot zugrunde liegt und es daher in den konkreten interpersonalen Handlungsbezügen aus sich heraus zu beschränken vermag“ (Hervorhebung L.R.).

77 Köhler (Fn. 45), S. 285, 670. Siehe ausführlich zur Differenzierung nach rechtlichen Verhältnissen und institutionellen Zusammenhängen - Familie, Gesellschaft, Staat - Köhler, in: Klesczewski u.a. (Hrsg.) (Fn. 54), S. 123 (133 ff.).

78 So auch Köbler (Fn. 45), S. 291. 
Kommt als Anknüpfungspunkt eine Verantwortlichkeit des Eingriffsadressaten beim Aggressivnotstand nicht in Frage, so kann der Eingriff nur auf dessen Solidaritätspflicht gestützt werden: Der Einzelne wird als Teil des allgemeinen Rechtsverhältnisses zu dessen Aufrechterhaltung herangezogen.

Im Folgenden (III.) wird auf die Grundzüge der Voraussetzungen einer solchen Notstandsinanspruchnahme eingegangen, ohne diese allerdings hier im Detail ausbuchstabieren zu können. ${ }^{79}$

\section{Voraussetzungen der Eingriffslegitimation aus dem Solidaritäts- bzw. Teilhabegedanken}

Die Voraussetzungen der Eingriffe nach dem erarbeiteten Prinzip rechtlicher Solidarität können gedanklich wie folgt strukturiert werden:

\section{Wesentliche Bedeutung des gefährdeten Rechtsguts für die Freiheitsverwirklichung}

Liegt der Grund des Notrechts in der Ursprungsgemeinsamkeit der Menschen, aus der sich ein Solidaritätsgebot dahingehend ergibt, die wesentlichen Voraussetzungen wechselseitiger Freiheit auch positiv zu gewährleisten, so können hieraus Anforderungen an die zu schützenden Güter des Notleidenden abgeleitet werden: Das Schutzgut muss die (nur partiell-ersetzlich, dazu unten) beeinträchtigen Rechtsgüter ${ }^{80}$ des Adressaten in seiner Bedeutung für die reale Freiheit - in der Terminologie des $\$ 34$ StGB - „wesentlich überwiegen“. Daher müssen auf der Seite des Notleidenden zwingend der Verlust des Lebens, schwere Einbußen an körperlicher Integrität oder persönlicher Freiheit zu befürchten sein.

Da nach dem hier dargelegten Eingriffsgrund das Anerkennungsverhältnis zum je Anderen durch die Not substantiell betroffen sein muss, kann es für die Annahme eines erheblichen drohenden Schadens entgegen Pawlik

79 Siehe insoweit auch die genannten Arbeiten von Helmers und Pawlik zum Notstand (freilich auf Grundlage ihrer je eigenen Ansätze); s. ausführlich zu den Eingriffsvoraussetzungen der Teilhabehaftung im Kontext des Strafverfahrens Rösin$\operatorname{ger}$ (Fn. 3), S. $171 \mathrm{ff} ., 176 \mathrm{ff}$.

80 Als „Rechtsgüter“ werden hier mit Zaczyk (Fn. 13), S. 165, 200 die „Daseinselemente der Freiheit" verstanden. Diesem zustimmend Pawlik (Fn. 2), S. 140, 162. Siehe dazu auch Köbler (Fn. 7), S. 33. 
nicht maßgeblich sein, ob bei der Addition der Interessen mehrerer in ihren Rechtsgütern bedrohter Personen die Bagatellgrenze überschritten ist. $^{81}$

Wann das „wesentliche Überwiegen“ nach diesem Verständnis konkret vorliegt, muss in dem jeweiligen Verhältnis und Kontext nach Prinzipien erarbeitet werden: In der hier thematischen unmittelbaren Not im zwischenmenschlichen Verhältnis geht es - wie dargelegt - um die Existenzbedingungen des Notleidenden. Im institutionellen Verhältnis (etwa im Kontext des Strafverfahrens ${ }^{82}$ ) geht es dagegen um die Aufrechterhaltung der Grundbedingungen der Staatlichkeit; Näheres muss im jeweiligen Sachzusammenhang erarbeitet werden. Jedenfalls kann die Voraussetzung des „wesentlich überwiegenden Eingriffsinteresses" nur in dieser prinzipiellen Bedeutung einen Platz in der Eingriffsrechtfertigung nach einem freiheitlichen Rechtsverständnis haben. ${ }^{83}$

An dieser Stelle, bei der Bestimmung des notwendigen Stellenwerts des Erhaltungsguts (auf Seite des Notleidenden), wird in einem weiteren Punkt der Wert der erläuterten Vorstrukturierung der Eingriffsrechtfertigung (gegenüber Unrechtsverantwortlichen bzw. Störern einerseits, gegenüber Unbeteiligten bzw. Nichtstörern andererseits) deutlich: ${ }^{84}$ Der häufig angeführte Maßstab, dass das von dem Eingriff betroffene „Interesse“ bzw. der durch den Eingriff verursachte Schaden nicht ,außer Verhältnis“ zum verfolgten Zweck stehen darf, ist nur anzuwenden, wenn der Eingriffsadressat für die Gefahr verantwortlich ist. Denn dann darf der Eingreifen-

81 Siehe Pawlik (Fn. 2), S. 266 ff. Kritisch dazu auch Neumann, ZStW 116 (2004), 751 (757).

82 Dazu ausführlich Rösinger (Fn. 3), S. 171 ff., insb. 176 ff.

83 An einer solchen Einordnung des „überwiegenden Interesses“ habe ich mich bereits in meiner Dissertation (Die Freiheit des Beschuldigten, 2019, S. 176 ff.) versucht. Allerdings habe ich mich dort, wie ich heute meine, zu sehr um eine (anknüpfungsfähige) Übersetzung in die Terminologie des Abwägungsgedankens bemüht. Meine dortigen Überlegungen zum Vorrangverhältnis zwischen Autonomie und Solidarität (S. 178) berücksichtigen den hier (oben unter II. 1.) dargelegten notwendigen Zusammenhang von Selbstbestimmung und Sozialität noch nicht ausreichend.

84 Dazu und zum Folgenden bereits Rösinger (Fn. 3), S. 178 f. 
de die Gefahr grundsätzlich zu Lasten des Verantwortlichen abwenden. ${ }^{85}$ Ausgeschlossen ist insoweit lediglich ein "grobes Missverhältnis“. ${ }^{86}$

Für den Aggressivnotstand bleibt festzuhalten: Das Tatbestandsmerkmal des „wesentlichen Überwiegens“ des Eingriffsinteresses hat in der Eingriffsrechtfertigung nach dem zugrunde gelegten Rechtsbegriff lediglich insoweit einen Platz, als es auf bestimmte Anforderungen an das gefährdete Rechtsgut des Notleidenden verweist: Dieses muss zur Freiheitsverwirklichung wesentlich sein. ${ }^{87}$

\section{Begrenzung auf partiell-ersetzliche Beeinträchtigungen}

Auch wenn derartige wesentliche Rechtsgüter durch die Not betroffen sind, ist nicht jeglicher Eingriff in die Rechtspositionen des Gegenübers, der zur Gefahrenabwendung notwendig (in der Terminologie des Verhältnismäßigkeitsgrundsatzes: geeignet und erforderlich) ist, automatisch rechtmäßig.

85 Siehe Puppe, Strafrecht, Allgemeiner Teil, 2011, S. 176 zu $\$ 228$; ebenso Erb, in: Joecks/Miebach (Hrsg.), Münchener Kommentar zum Strafgesetzbuch, 4. Aufl. 2020, $\$ 34$ Rn. 12 f. Es darf jedoch nicht übersehen werden, dass auch bei der Notwehr und dem Defensivnotstand nach $\$ 228$ BGB das Solidaritätsprinzip wirkt: Zwar begründet es dort nicht das Eingriffsrecht (dieses wird durch Verantwortung des Gegenübers begründet!), wohl aber beschränkt es das Eingriffsrecht auf Eingriffe, bei denen der Schaden nicht „außer Verhältnis“ zur abgewehrten Gefahr steht. Da beim Defensivnotstand die Rollen anders verteilt sind - der Unbeteiligte darf in Rechtsgüter des Verantwortlichen eingreifen -, handelt es sich auch dort um einen mittelbaren Eingriff in die Rechtssphäre des Unbeteiligten durch $B e$ schränkung seines Eingriffsrechts. Siehe dazu Kühnbach (Fn. 6), S. 89; Renzikowski (Fn. 5), S. 194 f.; Engländer, Grund und Grenzen der Nothilfe, 2008, S. 96.

86 Köbler (Fn. 7), S. 309. Dass auch beim Eingriff in die Rechte Unbeteiligter teilweise die Rede davon ist, die Beeinträchtigung dürfe nicht „außer Verhältnis“ zum Eingriffszweck stehen (siehe BVerfGE 27, 211, Rn. 16; in der StPO ausdrücklich in den $\iint 81$ II 2, 163 II 1, 121 I 2), zeigt nur, dass die notwendige Differenzierung nach Eingriffsgründen nicht bedacht wird (s. dazu mit Beispielen aus dem Strafverfahrensrecht genauer Rösinger [Fn. 3], S. 179 f., Fn. 336).

87 Dagegen kann der (o.g.) utilitaristische Ansatz, welcher Eingriffe nach dem Prinzip des überwiegenden Interesses begründet, die Forderung nach einem „wesentlichen Überwiegen“ nicht erklären; ein „einfaches“ Überwiegen müsste danach nämlich bereits reichen. Siehe dazu Pawlik (Fn. 2), S. 37 ff.; Helmers (Fn. 2), S. 57. 
Die weiteren Voraussetzungen ergeben sich aus dem Eingriffsgrund selbst: Die Beeinträchtigungen des Eingriffsadressaten dürfen nur partiell $(\operatorname{dazu} a))$ und ersetzlich $($ dazu $b))$ sein. ${ }^{88}$

\section{a) Opfergrenze}

Da durch den Notstandseingriff mit dem Erhalt der substantiellen Voraussetzungen der Personalität des Notleidenden das Verhältnis gegenseitiger Anerkennung gerade gewahrt werden soll, darf nicht auf der anderen Seite die Personalität des Eingriffsadressaten in Mitleidenschaft gezogen werden. Der Eingriff muss also nicht nur - auf Seiten des Notleidenden - der Gewährleistung der wesentlichen Voraussetzungen für eine Nutzung der Freiheit dienen, sondern er darf zugleich nicht den Adressaten in ebensolchen Voraussetzungen beschränken. Mit anderen Worten: Ein Mitkonstituent des Rechtsverhältnisses darf nicht seiner substantiellen Teilhabe daran beraubt werden. ${ }^{89}$ Die fortdauernde Teilhabe am allgemeinen Rechtsverhältnis ist also nicht nur Grund der Aufopferung, sondern auch deren Grenze. ${ }^{90}$ Die Teilhabe am allgemeinen Rechtsverhältnis setzt den darauf gestützten Eingriffen eine absolute Opfergrenze: ${ }^{91}$ Die Inanspruchnahme

88 Man kann die zusätzlich zu stellenden Anforderungen in Orientierung am geltenden Recht dem Terminus des „angemessenen Mittels“ ( $\$ 34$ S. 2 StGB) zuordnen (ohne dass durch diesen Begriff allein bereits etwas in der Sache ausgesagt wäre).

89 So Köhler, ZStW 107 (1995), 10 (23). Siehe dazu und zum Folgenden auch Rösin$\operatorname{ger}$ (Fn. 3), S. 184 ff., insb. $188 \mathrm{f}$.

90 Köhler (Fn. 7), S. 309. Zur legitimationstheoretischen Gebotenheit einer Aufopferungsgrenze beim Notstand auf Grundlage seines eigenen Ansatzes Pawlik (Fn. 2), S. 245 ff.: Die „eigenständige Personalität ,unschuldiger' Eingriffsopfer“ dürfe nicht negiert werden, da "die abstrakt-rechtliche Rechtsposition des Notstandspflichtigen (...) aufgehoben bleiben“ (i.S.v. erhalten bleiben) müsse (S. 248). Denn die „systematische Eigenart des abstrakten Rechts (erg.: als Garantie der Eigenständigkeit, L.R.) würde begrifflich in prinzipieller Weise negiert, wenn die Reichweite abstrakt-rechtlicher Positionen durchgängig relativ zu den Interessen anderer Personen bestimmt werden dürfte“ (S. 249). Deutlich S. 274: Die „rechtliche Verbundenheit" der Konfliktparteien dürfe nicht gesprengt werden.

91 Köhler (Fn. 7), S. 309 beschreibt diese als „Grenze unverrechenbar-substantieller Gutswahrung“. Dazu, dass die Opfergrenze nicht der „Interessenabwägung“ unverbunden gegenüberstehe, Pawlik (Fn. 2), S. 250: „Nach der hier entwickelten Auffassung stellt die Statuierung einer absoluten Aufopferungsgrenze nicht die äußerliche Beschränkung einer , an sich' umfassenden Interessenabwägung dar. Vielmehr trägt sie wesentlich mit dazu bei, den Raum zu konstituieren, innerhalb dessen die mit der Interessenabwägung zwangsläufig einhergehende Relativie- 
einer nicht spezifisch verantwortlichen Person darf sich nur auf partikuläre, nicht substantiell-erhebliche Aufopferungen erstrecken. ${ }^{92}$ Ausgeschlossen sind daher jedenfalls erhebliche Eingriffe in den Körper oder sonstige Freiheitsrechte. ${ }^{93}$ Nach einem dem hier vertretenen im Ausgangspunkt ähnlichen Ansatz soll der Notstand gänzlich auf Eingriffe in die Sachsubstanz beschränkt sein. So leiten Helmers ${ }^{94}$ und Köhler ${ }^{95}$ das Notrecht besitzrechtlich her: In der Not aktualisiere sich die „ursprüngliche Besitzgemeinsamkeit der Menschheit an der Weltsubstanz". ${ }^{96}$ Neben die einzig auf die Sachsubstanz beschränkte Pflicht im Notstand tritt allerdings für Köbler wegen der Annahme einer allgemeinen (aktiven) Hilfeleistungspflicht eine Befugnis zur Inanspruchnahme der allgemeinen Handlungsfreiheit in Not. ${ }^{97}$ Helmers lehnt auch eine solche Hilfspflicht ab, da er jeden Zugriff auf das „innere Mein“ ausschließen will. ${ }^{98}$ Diejenigen Fälle allerdings, in denen jemand eine andere Person (leicht am Körper verletzend bzw. nötigend) aus dem Weg stoßen muss, um sich selbst in Sicherheit vor einer

rung abstrakt-rechtlicher Positionen freiheitstheoretisch überhaupt nur akzeptabel ist.“ Ebenso steht nach Kühnbach (Fn. 6), S. 231 das „Opfermaximum“ außerhalb der Abwägung, weil es die Abwägung begrenze; ebenso wie das relevante Wohl (Eingriffsinteresse) müsse auch dieses Opfermaximum gesetzt werden als Rechtswert, der nicht geopfert werden muss; diese Absolutheit lasse sich nicht in die Abwägung einführen. So insbesondere Renzikowski (Fn. 5), S. 256 f.; Frister, GA 1988, 291 (293); Pawlik (Fn. 2), S. 96 f.; Schoch, JuS 1995, 34. Siehe auch Kühnbach (Fn. 6), S. 212 f. dazu, dass sich die Opfergrenze auch unmittelbar aus dem solidarischen Menschenbild in der Formulierung des BVerfG ergibt (vgl. BVerfGE 4, 7, Rn. 29).

92 So Köbler (Fn.7), S. 308 zum polizeilichen Notstand. Wann die betroffenen Rechtsgüter als für die Teilhabe „substantiell“ zu charakterisieren sind, muss erneut - wie auch bereits beim Schutzgut des Notleidenden (oben III. 1.) - im jeweiligen Sachkontext, differenziert nach institutionellen Verhältnissen erarbeitet werden. Siehe hierzu ausführlich für den Bereich staatlicher Eingriffe ggü. Nichtstörern überhaupt sowie speziell für den Strafverfahrenseingriff ggü. dem Beschuldigten Rösinger (Fn. 3), S. $184 \mathrm{ff}$.

93 So Köhler, ZStW 107 (1995), 10 (23). Ebenso zur Grenze beim Eingriffsadressaten im rechtfertigenden Notstand Pawlik (Fn. 2), S. 257 („Nur eine sachlich wie zeitlich beschränkte, nur eine punktuelle Beeinträchtigung seiner Lebensführung braucht er hinzunehmen").

94 Helmers (Fn. 2), S. 248 ff., 292 ff., 316.

95 Köhler (Fn. 45), S. 288 ff., insb. 290. Anders und weiter noch ders. (Fn. 7), S. 285 (der Eingriff könne sich „insbesondere“ auf das gegenständliche Eigentum beziehen), 290 (gerechtfertigt könnten „partikulär-unerhebliche Beeinträchtigungen auch der Körperintegrität und Freiheit anderer" sein).

96 Köhler (Fn. 45), S. 289.

97 Köhler (Fn. 45), S. 290.

98 Zusammenfassend Helmers (Fn. 2), S. 251. 
schweren (etwa Todes-)Gefahr zu bringen, will Helmers ebenfalls in die Notstandsrechtfertigung miteinbeziehen: Er sieht in diesen mittelbare Eingriffe in die Sachsubstanz. Der Notstandsadressat werde von dem Stück Boden weggestoßen, das der in Not Geratene gerade benötige. ${ }^{99}$ Diese Konstruktion, die auf den Sachsubstanzbezug besteht, wirkt auf mich etwas gekünstelt. Die Annahme weitergehender Eingriffsbefugnisse - sei es beim Notstand, sei es bei der allgemeinen Pflicht zur (aktiven) Hilfeleistung - erscheint mir nicht als zwingend mit der Fruchtbarmachung der Idee des Gesamtbesitzes für bestimmte Bereiche der Rechtfertigungsgründe unvereinbar: So kann zwar speziell für den Notstandseingriff betreffend die Sachsubstanz auf den Gedanken des ursprünglichen Gesamtbesitzes verwiesen werden; die Idee des Gesamtbesitzes ist jedoch lediglich ein Aspekt der Ursprungsgemeinsamkeit der Menschen, aus der (über vermittelnde Schritte, wie oben bei II. gezeigt) weitere Befugnisse und Pflichten im gegenseitigen Verhältnis für die Situation akuter Not hergeleitet werden können. ${ }^{100}$ Die Einordnung der leichten Körperverletzung oder der Beschränkung der allgemeinen Handlungsfreiheit, die auch im oben genannten Fall des Wegstoßens thematisch waren, als nur partielle und mit fortdauernder Teilhabe des Eingriffsadressaten noch vereinbare Aufopferungen erscheint mir daher auch abseits sachsubstanzbezogener Fälle nicht ausgeschlossen zu sein. ${ }^{101}$

\section{b) Anspruch auf Ausgleich}

Die Inanspruchnahme etwa des Eigentums des Unbeteiligten im Notstand soll verhindern, dass der Notleidende aufgrund des Verlusts seiner substantiellen Teilhabebedingungen aus dem Rechtsverhältnis ausscheidet und dieses damit erlischt. Daher kann sich der Eigentümer in der Not des Anderen nicht auf die ihm eigentlich zustehende Position berufen, wenn der

99 Etwa Helmers (Fn. 2), S. 315, Fn. 471.

100 Siehe auch Köhler (Fn.45), S. 265, dazu, dass Hilfeleistungspflicht und Notstandsrecht einen gemeinsamen Ursprung in dem (hinter dem Gedanken des ursprünglichen Gesamtbesitzes stehenden) Prinzip der Ursprungsgemeinsamkeit aller Menschen haben.

101 Dieser Unterschied in der Einordnung von über die Sachsubstanz hinausgehenden Beeinträchtigungen wird vor allem in institutionellen Verhältnissen relevant werden (derartige Eingriffe ablehnend Helmers [Fn. 2], S. 278 ff.), etwa bei der Beschuldigung im Strafverfahren (s. zum darin liegenden Eingriff Rösinger (Fn. 3), S. 48 ff., 153 ff., 189 ff.) oder - aktuell - im Falle von Ausgangsbeschränkungen und Ähnlichem anlässlich der Corona-Pandemie. 
Untergang des Rechtsverhältnisses selbst die Folge wäre. Dass der Eingriffsadressat aber endgültig die Last des Zufalls zu tragen hätte, ist damit nicht gesagt; er muss vielmehr Ausgleich erhalten: ${ }^{102}$ Der solidarisch Haftende wird zwar aus seiner - ihm nach Abgrenzung negativer Freiheitssphären eigentlich zustehenden ${ }^{103}$ - Position gedrängt, soll aber nicht von Rechts wegen ärmer werden. ${ }^{104}$ Daher müssen die Belastungen des Betroffenen vom Notstandsberechtigten wieder ausgeglichen werden. ${ }^{105}$ Der Notstand erlaubt keine dauerhafte „Umverteilung“ des Zufalls; die Einbuße darf im Hinblick auf einen Ausgleich nur vorläufig sein. ${ }^{106}$

102 Siehe zum Folgenden auch Rösinger (Fn. 3), S. $180 \mathrm{ff}$.

103 In der Diskussion um den rechtfertigenden Notstand wird unterschiedlich beurteilt, ob sich die Freiheitssphären durch Erlaubnisnormen gleichsam verschieben (so etwa Renzikowski, ARSP 2001, 119, Fn. 44; ähnlich ders., JRE 13 [2005], in: FS Hruschka, S. 643 [651]) oder ob der Eine ausnahmsweise zum Übergriff in die (fortbestehende) Sphäre des Anderen befugt ist (so wohl Köhler [Fn. 45] S. 284 f.). Siehe dazu auch Helmers (Fn. 2), S. 328, der sich gegen ein Regel-Ausnahme-Verhältnis wendet und die Notstandsregelungen als auflösende Bedingungen der Sondergebrauchsbefugnis einer Person hinsichtlich einer Sache im Verhältnis zu allen anderen ansieht. Der Aggressivnotstand bietet auch insoweit, als es um das Verhältnis des Tatbestands zur Rechtswidrigkeit geht, ein interessantes Feld für die Normentheorie. Die Diskussion kann insoweit hier nicht vertieft werden.

104 So Pawlik (Fn. 2), S. 9 unter Bezugnahme auf Löffler, ZStW 21 (1901), 537 (579). Zustimmend Kühnbach (Fn. 6), S. 234. Frister (Fn. 44), S. 31: Die Inanspruchnahme des Unbeteiligten unterliege unter anderem der Bedingung einer Entschädigung; dieser Ausgleichsgedanke finde sich in Art. 14 III GG sowie Art. 3 I GG (S.33) und trage dem Grundsatz der Lastengleichheit aller Bürger Rechnung (S. 34). Zum Zusammenhang zwischen Sonderopfergedanken und dem Prinzip der Pflichten- und Lastengleichheit auch Voßkuble, Das Kompensationsprinzip. Grundlagen einer prospektiven Ausgleichsordnung für die Folgen privater Freiheitsbetätigung, 1999, S. 25: Diese seien „aufs engste“ miteinander verbunden und wurzelten beide im Gleichheitssatz. Der Gesetzgeber sei „grundsätzlich gehalten, dort, wo er einzelnen Bürgern durch Gesetz besondere Pflichten auferlegt oder wo sie besondere Lasten zu tragen haben, die daraus resultierenden Nachteile zu kompensieren". Siehe ausführlich zur dogmatischen Einordnung des Ausgleichs bei staatlichen Eingriffen in die Rechtspositionen von Nichtstörern Rösinger (Fn. 3), S. 180 ff. m.w.N.

105 Siehe dazu einfachrechtlich $\$ 904$ BGB.

106 Siehe dazu Köhler (Fn. 7), S. 290 zum rechtfertigenden Notstand. 


\section{Schluss}

Die dargelegte rechtsprinzipielle Unterscheidung zwischen der Inanspruchnahme eines für die Not Verantwortlichen einerseits und eines insoweit Unbeteiligten andererseits, die durch ein bloßes Abwägen von Interessen verwischt zu werden droht, zeigt ihre Bedeutung gerade in krisenhaften Situationen wie der derzeitigen ${ }^{107}$ Lähmung weiter Teile des öffentlichen Lebens durch die Maßnahmen gegen die Corona-Pandemie. Im aktuellen Zusammenhang ist die Rede von Solidarität allgegenwärtig, ohne dass immer ganz deutlich würde, ob es um einen moralischen Appell an das Zusammengehörigkeitsgefühl, um ein Werben um das Verständnis der von beschränkenden Maßnahmen Betroffenen oder um eine Forderung des Rechts geht.

Für den Gegenstand der hiesigen Untersuchung, den Eingriffsgrund des Aggressivnotstandes, wurde die Solidarität als Rechtsbegriff eingeführt. Eine grenzenlose Inanspruchnahme von für die Not nicht verantwortlichen Personen ist hiermit nicht begründbar. Die dargelegten Grundbestimmungen sollten erinnert werden, wann immer durch die Politik schwerwiegende Eingriffe unter nicht weiter begründetem Verweis auf den Zusammenhalt der Gesellschaft oder gar mit ihrer bloßen Notwendigkeit zum Schutz eines vermeintlich „überwiegenden Interesses“ zu rechtfertigen gesucht werden. 
